\title{
Comparison of immunofluorescence and enzyme linked immunosorbent assays for diagnosing Lyme disease
}

\author{
S J CUTLER, D J M WRIGHT Department of Medical Microbiology, Charing Cross Hospital, London
}

SUMMARY A new enzyme linked immunosorbent assay (ELISA) method was assessed in parallel with an indirect immunofluorescence assay (IFA) for the serological testing of specimens submitted for detection of Lyme disease. Wells of an ELISA microtitre plate were coated with sonicated whole cells of Borrelia burgdorferi in carbonate buffer. After overnight incubation at $4{ }^{\circ} \mathrm{C}$ the plates were washed three times, then incubated at $37^{\circ} \mathrm{C}$ for an hour sequentially with a blocking solution, diluted test serum, anti-human IgG horseradish peroxidase conjugate, and finally for half an hour at room temperature with o-phenylenediamine substrate before being read at $492 \mathrm{~nm}$. Absorbance results were converted into arbitrary ELISA units. Of a total of 1760 sera, $146(8.3 \%)$ were positive: of these $92(63 \%)$ were positive by both methods, five (3.4\%) by IFA alone, and $49(33.6 \%)$ by ELISA alone. The ELISA was better suited for testing large numbers of specimens and easier to interpret than IFA.

Lyme disease is a multisystem disorder caused by a tick borne spirochaete, Borrelia burgdorferi. The presenting clinical manifestations are diverse, ranging from skin lesions and non-specific flu-like symptoms to carditis, neuritis, and arthritis. ${ }^{1}$ Laboratory confirmation of the clinical diagnosis by conventional microscopy and culture methods has been largely unsuccessful. This has led to the development of serological tests for the diagnosis of Lyme disease. ${ }^{2}$

The indirect immunofluorescence assays (IFA) were the first tests to become established. The test is, however, subjective and difficult to automate. The IFA is being gradually superseded in the United States of America by the enzyme-linked immunosorbent assays (ELISA) as it is more sensitive and specific. ${ }^{3-5}$ We now report a new ELISA method for the detection of Lyme disease antibodies and compare it with IFA using all sera submitted over a consecutive nine month period (December 1987 to August 1988).

\section{Material and methods}

A total of 1760 sera submitted for Lyme disease serology were tested in parallel by both IFA and ELISA methods. To assess cross reactivity, we examined sera from patients with primary, secondary, and tertiary syphilis $(n=18)$ and sera drawn from patients at one, seven, and 14 days after diagnosis of louse born relapsing fever $(n=16)$. Sera from patients with active collagen disorders $(n=17)$ and with raised titres against Epstein-Barr virus (EBV) $(n=15)$ were also tested. The EBV titres were Accepted for publication 14 February 1989 established using a standard immunofluorescence method with infected HR1K cells used as antigen. Titres ranged from 5 to 1280 . Lastly, as control sera, antenatal specimens were obtained from both rural (Hampshire) $(\mathrm{n}=132)$ and urban (London) $(\mathrm{n}=$ 158) populations.

Antigen was prepared from the B31 reference strain of $B$ burgdorferi grown in modified BSK medium for seven days at $33^{\circ} \mathrm{C}$. Cultures were harvested and washed with 5 volumes of phosphate buffered saline (PBS) (pH 7·3, Oxoid) using a Minitan ultrafiltration system (Millipore).

\section{IMMUNOFLUORESCENCE ASSAY}

Multi-well immunofluorescence slides (Flow) were coated with $20 \mu \mathrm{l}$ per well of borreliae suitably diluted in PBS to give a semiconfluent covering of spirochaetes. Air dried slides were fixed in $10 \%$ methanol in distilled water for two minutes, dried, and stored at $-20^{\circ} \mathrm{C}$ until required. Test sera diluted to $1 / 64$ and 1/256 in PBS was applied to antigen coated wells in $20 \mu \mathrm{l}$ volumes. After 30 minutes of incubation at $37^{\circ} \mathrm{C}$ in a moist chamber the slides were washed with two five minute changes of PBS. Anti-human IgG fluorescein isothiocyanate-labelled conjugate (Wellcome) diluted 1/60 in PBS was then layered on to wells in $20 \mu \mathrm{l}$ volumes and the slides incubated and washed as before. Air dried slides were mounted and read using an Ortholux fluorescence microscope with a $\times 50$ water immersion Leitz objective. Titres were expressed as the reciprocal of the highest dilution of serum giving definite fluorescence $(++$ on a scale of + to ++++ ), of at least $50 \%$ of spirochaetes. 
Specimens showing fluorescence were retested at five doubling dilutions from $1 / 64$ to $1 / 1024$, and reported positive if the titre was 256 or above.

\section{ELISA}

Antigen was sonicated while cooled on ice, for six 15 second bursts at $60 \%$ of the maximum intensity of a sonicator (MSE), and $0.01 \%$ aqueous thimerosal added.

Protein concentration was determined according to the manufacturer's instructions based on the Bradford method (Bio-Rad). The optimal antigen concentration, determined by titration (usually $10 \mu \mathrm{g} / \mathrm{ml}$ ), was diluted in $0.05 \mathrm{M}$ carbonate-bicarbonate buffer $(\mathrm{pH}$ 9.6), prior to coating wells of flat-bottomed Linbro microtitre plates (Flow). Alternate wells were coated with $100 \mu \mathrm{l}$ volumes of diluted antigen (test wells), the remaining wells received $100 \mu \mathrm{l}$ of coating buffer (control wells). After overnight incubation at $4{ }^{\circ} \mathrm{C}$ plates were washed three times with PBS containing $0.05 \%$ of Tween 20 (Sigma). Non-specific binding sites were blocked by a one hour incubation at $37^{\circ} \mathrm{C}$ with $0.5 \%$ horse serum in PBS (200 $\mu \mathrm{l}$ per well). Plates were washed as before and $100 \mu$ l volumes of test serum diluted $1 / 300$ in PBS-Tween 20 containing 5\% horse serum (serum diluent), added to both test and control wells. After one hour of incubation plates were again washed, and $100 \mu \mathrm{l}$ of anti-human IgG conjugated with horse radish peroxidase (ICN) diluted $1 / 2000$ in serum diluent added to each well. After a further hour of incubation and washing, $100 \mu \mathrm{l}$ of ophenylenediamine substrate $(4 \mathrm{mg} / \mathrm{ml}$, Sigma) in $0.1 \mathrm{M}$ citrate-phosphate buffer (pH5), activated with $0.02 \% \mathrm{H}_{2} \mathrm{O}_{2}$, was added to each well and the plates incubated for 30 minutes at room temperature in the dark. The enzymatic reaction was stopped by the addition of $100 \mu \mathrm{l}$ of $1 \mathrm{~N} \mathrm{H}_{2} \mathrm{SO}_{4}$, and plates read at $492 \mathrm{~nm}$ on a Titertek Multiskan (Flow).

Positive and negative control sera were included on every plate. Test plates giving $0 \cdot 1$ absorbance units difference from the expected negative control value $(+/-5 \%)$, and 0.2 absorbance units difference from the positive value $(+/-10 \%)$, were rejected.

Control absorbance readings were deducted from the test well values and results converted into arbitrary units using a standard curve of absorbance of pooled positive sera plotted against ELISA units (fig 1). Results above 20 units were considered to be positive (the positive control serum had a value of 100 units). Those results under 30 units were reported as weak positives. Results between 10 and 20 units were considered to be equivocal, but reported as negative.

Results were compared using McNemar's $\chi^{2}$ test.

\section{Results}

Of the 1760 sera submitted for Lyme disease serology,

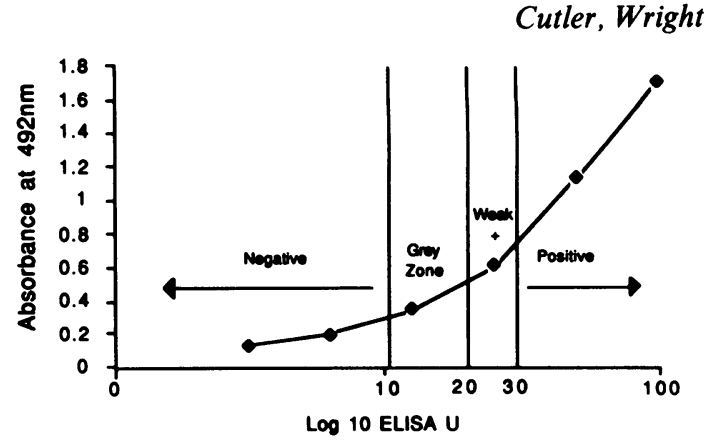

Fig 1 Conversion of ELISA absorbance readings into units.

$146(8 \cdot 3 \%)$ were positive by at least one method (fig 2). If IFA was used alone, only $97(66.4 \%)$ positive results would have been detected; ELISA alone would have detected $141(96.6 \%)$ of the positive results. The ELISA yielded significantly more positive results (McNemar's $\chi^{2} 34 \cdot 24, \mathrm{p}=<0.001$ ) than IFA. Of the positive results, $20.5 \%$ were previously positive patients, $13 \%$ gave a history of tick or "insect" bite, $22.6 \%$ had skin lesions, $11.6 \%$ flu-like illness, $2 \%$ cardiac complications, $17.8 \%$ neurological manifestations and $16.4 \%$ joint symptoms.

Overall agreement between both methods for positive and negative results was $95.6 \%$ (fig 2), with a correlation coefficient of 0.999 .

Three of the 146 sera positive for Lyme disease also had positive syphilis serology. Of these one was strongly positive in Lyme serological tests, one equivocal by both methods, and the remaining one was equivocal by IFA and negative by ELISA $(<10$ units). Patients with syphilis and relapsing fever showed cross reactivity with both ELISA and IFA (table). This was greatest in patients with acute disease. Of patients with EBV antibodies, only one of 15 specimens was positive (by both methods, EBV titre 80 ); seven of these specimens gave equivocal results. No association was found between EBV titre and cross reactivity in serological tests for Lyme disease. Patients with active collagen disorders also produced occasional false positive results, unrelated to titre in

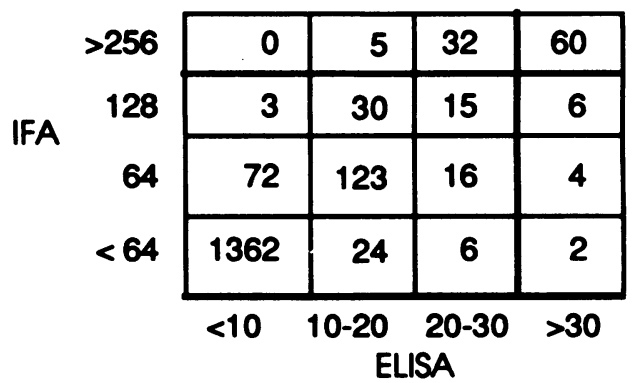

Fig 2 Distribution of IFA and ELISA results. 
Table Positive results detected with different groups of sera

\begin{tabular}{lrrlr}
\hline Group & No & Both & IFA & ELISA \\
\hline Routine & 1760 & 92 & 5 & 49 \\
Syphilis & 18 & 10 & 0 & 2 \\
Relapsing fever & 16 & 3 & 0 & 10 \\
EBV & 15 & 1 & 0 & 0 \\
Collagen disease & 17 & 0 & 0 & 2 \\
Urban ANC* & 158 & 1 & 1 & 0 \\
Rural ANC* & 132 & 1 & 0 & 9 \\
Total & 2116 & 108 & 6 & 72 \\
\hline
\end{tabular}

* = Antenatal control sera.

either antinuclear factor or rheumatoid factor tests. Healthy negative controls from rural and urban areas were $7.6 \%$ and $0.6 \%$ positive by ELISA, respectively, and $0.8 \%$ and $1.3 \%$ positive by IFA. None of the urban area positive results had ELISA units greater than 30 , and only $3 \%$ of rural dwellers gave results greater than 30 units.

\section{Discussion}

The problems encountered during serological testing for Lyme disease have recently been reviewed. ${ }^{2}$ This study confirms that IFA and ELISA methods are of comparable specificity. ${ }^{3}$ Our data show that ELISA gives more positive results, ELISA yielding $96.6 \%$ of positive results while only IFA detects $66.4 \%$ of the positive specimens.

The specificity of serological tests for Lyme disease requires further work. Cross reactivity of serum from patients with other spirochaetoses and acute phase collagen disorders has been documented,,$^{2-4}$ and was shown in this study. Many cross reacting sera gave equivocal results but few gave positive results. Early cases of Lyme disease and treated cases may also give equivocal results. The IgG response in Lyme disease rises only slowly, ${ }^{2}$ not reaching significance until the fourth week. Our practice is to ask for a further specimen from patients giving equivocal results, as well as requesting other tissue fluids as appropriate. The presence of antibodies to $B$ burgdorferi in these fluids is usually detected using the IFA and ELISA.
Specific IgM class antibodies may be helpful in determining whether early infection has occurred.

It is essential to exclude potential false positive results by testing all positive sera for syphilis and autoantibodies. Western blot analysis may be required to assist in the interpretation of positive serological results.

It is recommended that the ELISA method should be used for serological screening because of its increased sensitivity, lack of subjectivity and ease of automation. The relevance of positive results with the clinical diagnosis may require Western blotting to resolve problem cases.

We thank the following in our department: Amanda L Colebrook, Anthony Fairbairn, Laurence A Willis and Ralph Dayton for their assistance. We also thank Dr KD McRae (Reader in Medical Statistics, Charing Cross Hospital) for statistical advice. We are especially grateful to Dr S Patrick (PHLS Odstock) for providing the antenatal sera from Hampshire.

This work was supported by a grant from North West Thames Region Health Authority.

\section{References}

1 O'Neill PM, Wright DJM. Lyme disease. Br J Hosp Med 1988; 40:284-9.

2 Cutler SJ, Wright DJM. Serodiagnosis of Lyme disease. Serodiagn Immunother Infect Dis 1988;2:4-6

3 Craft JE, Brodzicki RL, Steers AC. Antibody response in Lyme disease: evaluation of diagnostic tests. J Infect Dis 1984; 149:789-95.

4 Russell H, Sampson JS, Schmid GP, Wilkinson HW, Plikaytis B. Enzyme-linked immunosorbent assay and indirect immunofluorescence assay for Lyme disease. $J$ Infect Dis 1984;149: 465-70.

5 Magnarelli LA, Meegan JM, Anderson JF, Chappell WA. Comparison of an indirect fluorescent-antibody test with an enzymelinked immunosorbent assay for serological studies of Lyme disease. J Clin Microbiol 1984;20:181-4.

Requests for reprints to: Dr Sally J Cutler, Research Assistant, Microbiology Department, Charing Cross Hospital, Fulham Palace Road, London W6 8RF, England. 